\title{
Metástasis coroidea como debut de un carcinoma de pulmón no microcítico avanzado
}

\author{
E. Villar Chamorro, M. Cobo Dols, S. Gil Calle, A. Montesa Pino, J. Alcaide García, I. Alés Díaz, \\ V. Gutiérrez Calderón, G. Durán Ogalla
}

\section{Resumen}

Presentamos la descripción del caso clínico de un paciente de 65 años con un carcinoma de pulmón no microcítico (CPNCP) avanzado que debuta con una alteración del campo visual derecho, sin otros síntomas, diagnosticándose en la exploración oftalmoscópica de metástasis coroidea, con la realización de una angiografía fluresceínica para diferenciarlo de una hemorragia o un hemangioma. Este caso destaca por el área infrecuente de metástasis y la inusual forma de presentación de este tipo de tumor.

\section{Palabras clave:}

Metástasis coroidea. Carcinoma pulmón no microcítico. Oftalmoscopia.

Oncología, 2006; 29 (1):43-46 


\section{Summary}

We present the clinical case of a 65 year old patient with right visual field disturbance as debut of a disseminated non-small cell lung cancer (NSCLC), without any other symptom. Ophthalmoscopy detected choroidal metastases, that fluorescein angiography delimited and differentiated from hemorrhage and hemangioma. The case emphasizes the infrequent production of metastases in this area by this tumor, and, above all, the unusual clinical debut.

Key words: Choroid metastases. Non-small cell lung cancer. Ophthalmoscopy.

\section{Presentación del caso}

Se trata de un paciente varón de 65 años, fumador de 40 paquetes/año, hipertensión arterial con buen control farmacológico, miopía e intervenido de catarata ojo derecho 5 años antes, como antecedentes más destacables. El motivo de consulta en el Servicio de oftalmología de nuestro hospital, consistió en un cuadro de un mes de evolución de un escotoma no central en el ojo derecho. No presentó síntomas respiratorios, ni cuadro constitucional.

En la exploración oftalmoscópica se identificaba en la porción más superior de la papila, una imagen de despigmentación, con áreas pigmentadas anaranjadas en el centro, de un tamaño de 4-5 diámetros papilares y levantamiento exudativo de los bordes (Figura 1). Se realiza angiografía fluoresceínica en la que se reconoce de nuevo esta misma lesión des-

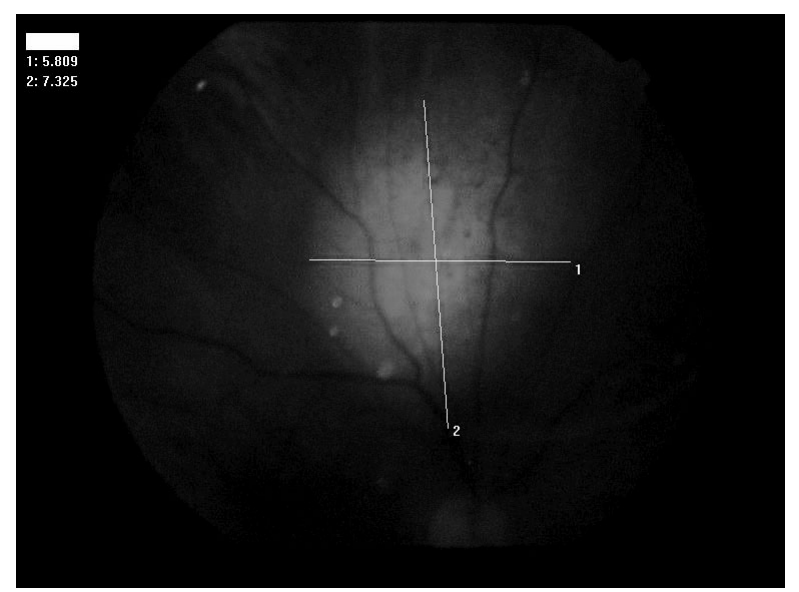

Figura 1. Retinografía en la que se aprecia en la porción más superior de la papila la imagen de metástasis coroidea.

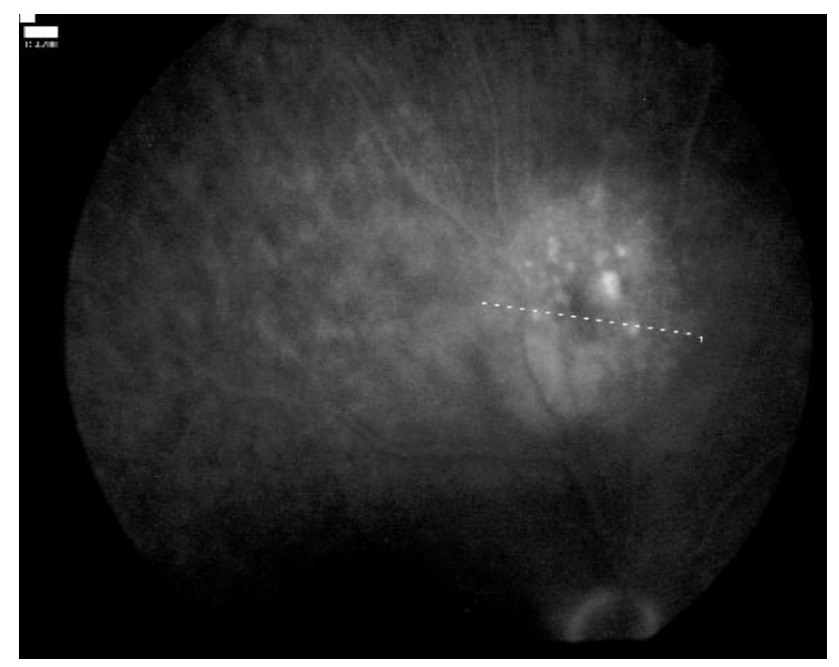

Figura 2. Angiografía fluresceínica, en la que se aprecia la imagen de metástasis coroidea.

crita en la retinografía, y se descartó de un hematoma o hemangioma (Figura 2). La ecografía orbitaria mostraba una masa de 7 × 3 milímetros $(\mathrm{mm})$ de diámetro, con una elevación de $3 \mathrm{~mm}$. Se realiza una resonancia magnética nuclear de la órbita, en la que se aprecia un mínimo engrosamiento focal retiniano inmediatamente adyacente y lateral a la papila, sin hiperseñal ni realce tras la administración de contraste. Un mes después se realiza una nueva retinografía y ecografía orbitaria, en la que se aprecia un claro aumento de la lesión retiniana, llegando a una dimensión de 9,6 x 3,9 mm. La aproximación diagnóstica fue de tumor retiniano, posiblemente metastásico.

En el estudio general de búsqueda del tumor primario, se realiza una tomografía axial computeriza- 
da (TC) toracoabdominopélvica en la que se aprecia en lóbulo superior izquierdo un nódulo de $2,2 \mathrm{~cm}$, que engloba la salida del bronquio del lóbulo superior, así como adenopatías mediastínicas de tamaño superior a 2 centímetros $(\mathrm{cm})$ prevasculares, pretraqueales derechas, ventana aortopulmonar y subcarinales. A nivel abdominal destacan nódulos suprarrenales bilaterales de $2 \mathrm{~cm}$ en el izquierdo y $1,5 \mathrm{~cm}$ en el derecho. La Tomografía por emisión de positrones (PET) mostraba múltiples focos de elevada actividad metabólica a nivel paratraqueal derecho, mediastínico y región hiliar y parahiliar izquierdo, región suprarrenal derecha, todo ello compatible con afectación tumoral maligna. Se realiza mediastinoscopia para toma de biopsia de una adenopatía paratraqueal derecha, cuyo resultado fue metástasis de adenocarcinoma.

Con el diagnóstico de metástasis retiniana derecha y suprarrenal de adenocarcinoma de pulmón, comienza tratamiento quimioterápico con esquema docetaxel y cisplatino, mostrando tras dos ciclos una respuesta parcial de la enfermedad medible y una aparente mejoría de la formación retiniana, aunque posteriormente, se complicó con un desprendimiento de retina leve y tuvo que ser tratado con radioterapia.

\section{Discusión}

Se desconoce exactamente la incidencia de las metástasis de tumores sólidos o hematológicos en el globo ocular, pero se estima que es superior a la de tumores primarios, básicamente el melanoma. La localización mas frecuente es la coroides y, en general, son raras y se suelen diagnosticar en pacientes en estadios tumorales muy avanzados, por lo que la mayoría de las series publicadas son descriptivas de necropsias ${ }^{1,2}$.

Según los datos obtenidos de la combinación de varias series, los tumores primarios se localizaban en la mama en el $47 \%$ de los casos, en el pulmón en el 25\%; riñón y tracto gastrointestinal en el $3 \%$ respectivamente, testículos en el $2 \%$, y por último, una miscelánea de tumores de próstata, páncreas, tiroides y melanoma cutáneo, en el $1 \%$. En el $15 \%$ de los casos, la localización del tumor primario es desconocida. Por tanto podemos observar que la detección metástasis coroideas es más frecuente en los pacientes afectos de cáncer de mama y pulmón, aunque no sea esta la localización más frecuente de la enfermedad diseminada, ocupando en el caso del cáncer de pulmón un $6^{\circ}$ lugar ${ }^{3-5}$.

Las metástasis oculares pueden producirse a cualquier edad pero son mas frecuentes en pacientes de edades comprendidas entre 40-70 años. Se ha encontrado una clara diferencia entre los pacientes con cáncer de pulmón con o sin metástasis, siendo los pacientes jóvenes los que tienen mayor incidencia de afectación ocular, lo que sugiere que la edad temprana podría constituir un factor de riesgo ${ }^{6}$.

Los signos y síntomas de presentación ocular son variables dependiendo del tamaño, la localización y los efectos secundarios que produzca el tumor. La visión borrosa y el dolor ocular suelen ser los síntomas mas frecuentes. La complicación que se puede presentar con más frecuencia es el desprendimiento de retina. La existencia de metástasis coroideas es un signo de mal pronostico pues el tiempo de supervivencia medio tras su diagnostico es corto ${ }^{7}$.

Es bien conocido el aspecto amarillo cremoso que adoptan las metástasis coroideas cuando se ven con el oftalmoscopio, aunque en las metástasis de mayor tamaño el color es más anaranjado (Figura 1). La angiografía fluoresceínica (Figura 2) ayuda a delimitar la lesión y a diferenciar de hemorragias y lesiones vasculares como los hemangiomas, pero raramente permitirá discernir claramente de otras lesiones tumorales coroideas como los melanomas amelanóticos. Otras formas de diagnostico será el uso de la ultrasonografia, la resonancia magnética nuclear y la realización de PAAF para el diagnostico histológico en el caso de tumores de origen desconocido $^{2}$.

En cuanto al tratamiento se puede observar que la quimioterapia no consigue respuesta a nivel coroideo en la mayor parte de los pacientes, siendo el tratamiento de elección la radioterapia, con la que se consigue el control sintomático de los pacientes ${ }^{7}$.

El caso que presentamos se trata de un CPNCP avanzado que debuta como primera manifestación con una alteración del campo visual, y este fue el signo guía que llevó al diagnóstico del tumor. Cabe destacar lo inhabitual de esta forma de presentación, al ser la localización coroidea una diana de invasión tumoral en fases muy evolucionadas de la enfermedad neoplásica. En nuestro conocimiento, sólo ha 
sido descrito en la literatura un caso de similares características en cuanto a la forma de presentación ${ }^{8}$. Por otro lado, aunque el tratamiento de elección de esta entidad es la radioterapia ocular, en nuestro caso se optó por comenzar con quimioterapia, debido al componente avanzado del tumor, y se trató con radioterapia tras manifestar la complicación del desprendimiento de retina.

Correspondencia:

Dr. M. Cobo Dols

Sección de Oncología Médica

Pabellón A, $3^{\circ}$ planta, Derecha

Secretaría de Oncología Médica

Hospital Regional Universitario Carlos Haya

Avda. Carlos Haya, s/n

E-29010 Málaga

manuelcobodols@yahoo.es

mangel.cobo.sspa@juntadeandalucia.es

\section{Bibliografía}

1. Jeddi A, Bouguila H, Mezlini A, Daghfous F, Kaoueche M, Ben Ayed F, et al. Choroid Metastases. J Fr Ophtalmol 1994;17(11):657-63.

2. De Potter P, Disneur D, Levecq L, Snyers B. Ocular manifestations of cancer. J Fr Ophtalmol 2002 ;25(2) :194-202.

3. Da Silva Correa ZM, Burmann TG, Moraes Freitas A, Zatti Ramos G, Mundialino Marcon I. Prevalence of ocular metastasis in patients with known metastatic disease: preliminary results. Arq Bras Oftalmol, Vol 68 n², Sao Paulo Mar/Apr.2005.

4. Vidal Candela MT, Javaloy Estaño J, Soler Ferrández FL, Rodríguez-Lescure A, Carrato Mena A. Metástasis coroideas en el cáncer de mama y pulmón. Prevalencia y características clínicas. Estudio prospectivo. Arch. Sociedad Española Oftalmología 2002, Nº1 Enero.

5. Kreusel KM, Wiegel T, Stange M, Bornfeld N, Foerster $\mathrm{MH}$. Intraocular metastases of metastatic breast carcinoma in the woman. Incidence, risk factors and therapy. Ophthalmologe 2000 May; 97 (5):342-6.

6. Kreusal KM, Wiegel T, Stange M, Bornfeld N, Hinkelbein $\mathrm{W}$, Foerster MH. Choroidal metastases in disseminated lung cancer: frecuency and risk factors. Am J Ophthalmol 2002; 134(3):445-7.

7. Sassmannshausen J, Bornfeld N, Foerster MH, Sauerwein W, Schreiber T, Wessing A. Metastases of malignant extraocular tumors to the choroids. Diagnosis and fractionated radiotherapy. Fortschr Ophthalmol.1990;87(1):69-73.

8. Abraldes López-Veiga MJ, Mesa Lugo FI, Arteaga Hernández VJ, Cordoves Dorta LM, Pérez Barreto L, Serrano García MA. Metástasis coroidea como primera manifestación de Adenocarcinoma en pulmón. Arch Soc Canar Octal. 2001-Nº12. Caso clínico. 